patients. In addition, about 11 cases of mild to moderate dysplasia were identified.

Conclusions Early identification and curative resection still seem to be the best bet for a successful outcome in gallbladder cancer. Incidental gallbladder cancer should be suspected in elderly patients with thickened Gall Bladder. Imaging forms a very important component of preoperative diagnosis. Patients with $\mathrm{T}$ stage of $\mathrm{T} 1 \mathrm{~b}$ and above should be offered reresection to give the advantage of improved survival.

\section{IDDF2018-ABS-0046 DIRECT ACCESS ENDOSCOPY BOOKING BY FAMILY PHYSICIANS: EVALUATING A NEW SERVICE MODEL AND CLINICAL PREDICTORS OF POSITIVE ENDOSCOPY FINDINGS AT PRIMARY CARE SETTING}

Lok Hang Leung*. Department of Family Medicine and Primary Health Care, Kowloon West Cluster, Hospital Authority, Hong Kong

\subsection{6/gutjnl-2018-IDDFabstracts.82}

Background Dyspepsia is a common clinical problem in the Asia-Pacific region and can have a variety of presentations. Patients who have dyspepsia and need an oesophagogastroduodenoscopy (OGD) are referred by their primary care doctor to surgeons or gastroenterologists, who would perform the OGD. In order to shorten the waiting time for patients indicated for an endoscopy (GOPC-to-endoscopy time), a mode of open-access was first available in HK in the 1990s. At the Kowloon West Cluster (KWC), five GOPCs implemented direct access endoscopy since late 2015 in collaboration with Department of Surgery, Caritas Medical Centre. OGDs, which were performed by designated surgeons, were arranged by GOPC doctors directly. Post-OGD follow-up care would be offered by GOPC.

Objectives To evaluate direct access endoscopy model and GOPC-to-endoscopy waiting time.

To review endoscopic outcomes of patients under direct access endoscopy programme.

To identify clinical predictors for positive OGD findings for patients presenting at primary care setting.

Methods A retrospective cohort study from 1 st Oct 2015 to 31st Dec 2016. Adult patients who had OGD booked under the programme were included. Demographics and clinical characteristics variables were compared. Variables with $p<0.1$ in the simple logistics regression analysis were included in the multiple regression models. Adjusted odds ratio and 95\% CI were calculated. A P-value of $<0.05$ was considered statistically significant.

Results 198 patients were arranged direct access endoscopy. 173 patients completed OGD. The mean GOPC-to-endoscopy time was 14 weeks $(23.7 \%$ within 8 weeks). 26 patients had positive OGD findings including ulcer and neoplastic conditions, one of which was stomach adenocarcinoma. Clinical predictors for a positive OGD included ever smoking status (adjusted OR 3.15; 95\% CI 1.00-9.86; P 0.049), presence of epigastric pain on history (adjusted OR 3.32; 95\% CI 1.199.26; P 0.022) and a positive $\mathrm{H}$ Pylori status (adjusted OR 3.60; 95\% CI 1.39-9.36; P 0.009).

Conclusions Direct assess endoscopy in primary settings may have a role in early detection of significant pathologies. Clinical predictors may be useful for triage purpose as the patient may not present classical red flags symptoms in primary settings.

\section{IDDF2018-ABS-0047 ACUPUNCTURE AND RELATED THERAPIES FOR TREATING IRRITABLE BOWEL SYNDROME: OVERVIEW OF SYSTEMATIC REVIEWS AND NETWORK META-ANALYSIS}

${ }^{1}$ Charlene Hoi Lam Wong* ${ }^{2}$ Irene Xin Yin Wu, ${ }^{3}$ Robin Sze Tak Ho, ${ }^{3}$ William Kwok Wai Cheung, ${ }^{4}$ Alexander C Ford, ' ${ }^{1}$ Justin Che Yuen Wu, ${ }^{5}$ Holger Cramer, ${ }^{3}$ Samuel Yeung Shan Wong, ${ }^{3}$ Vincent Chi Ho Chung. 'Department of Medicine and Therapeutics, The Chinese University of Hong Kong, Hong Kong: ${ }^{2}$ Hong Kong Institute of Integrative Medicine, The Chinese University of Hong Kong, Hong Kong; ${ }^{3}$ Jockey Club School of Public Health and Primary Care, The Chinese University of Hong Kong, Hong Kong; ${ }^{4}$ Leeds Gastroenterology Institute, St. James's University Hospital, Leeds, UK; ${ }^{5}$ Department of Internal and Integrative Medicine, University of Duisburg-Essen, Germany

\subsection{6/gutjnl-2018-IDDFabstracts.83}

Background Although existing clinical evidence has suggested potential effectiveness of acupuncture and related therapies for improving irritable bowel syndrome (IBS) symptoms, comparative effectiveness of different acupuncture modalities is unclear. An overview of systematic reviews (SRs) and a network metaanalysis (NMA) were conducted to evaluate the comparative effectiveness of acupuncture and related therapies used either alone, or as an add-on to other IBS treatments.

Methods Eight international and Chinese databases were searched for SRs of randomised controlled trials (RCTs) that investigated the effectiveness of acupuncture and related therapies among IBS patients. Data from eligible RCTs were extracted for random effect pairwise meta-analyses. NMA was used to evaluate the comparative effectiveness of different treatment options.

Results From 15 SRs, 28 RCTs $(n=2314)$ assessing acupuncture and related therapies used either alone or as an add-on to other IBS treatments were included. Results from pairwisemeta-analysis showed that needle acupuncture was superior in improving global IBS symptoms, compared with both pinaverium bromide and trimebutine maleate. Electroacupuncture was found to have significantly stronger effects in alleviating global IBS symptoms when compared with pinaverium bromide. Needle acupuncture plus moxibustion was significantly more effective than loperamide in improving global IBS symptoms. Significant add-on effect was also observed in the pooled results of needle acupuncture plus Chinese herbal medicine (Geshanxiaoyao formula) when compared with Geshanxiaoyao formula alone. Results from NMA showed a combination of needle acupuncture and Geshanxiaoyao formula had the highest probability of being the best option for improving global IBS symptoms among 14 included treatment options. No serious adverse events associated with acupuncture and related therapies were reported.

Conclusions Amongst reviewed treatment options, the combination of needle acupuncture and Geshanxiaoyao formula had the highest probability of being the most effective treatment for global IBS symptoms. Patients who are contraindicated for current conventional pharmacological or non-pharmacological therapies may use acupuncture and related therapies. Future SRs should investigate the potential combination effect of other Chinese herbal medicine plus acupuncture and related therapies for treating IBS. 